\title{
Probiotics in health and disease
}

\author{
Deepinder Kaur ${ }^{1}$, Rajoo Singh Chhina ${ }^{2}$ \\ Department of Microbiology ${ }^{l}$, Department of Gastroenterology ${ }^{2}$ \\ Dayanand Medical College and Hospital, Ludhiana, Punjab
}

Proviotics, the term that literally means "for life" was first introduced in 1953 by Werner Kolkith. Russian Nobel Laureate Elie Metchnikoff, proposed the idea that ingesting microbes could have beneficial effects for human beings. The modern definition drafted by the Joint expert consultation of the Food and Agricultural Organization of the United Nations and the World Health Organization in 2001 defined probiotics as "live microorganisms, which when consumed in adequate amounts, confer a health benefit on the host". Studies of metagenomics and the human microbiome will expand the knowledge on the composition of microbiota of the humans. The speciality of gastroenterology will be unanimously benefited from the ability to rationally modify the microbiota with the use of antibiotics, probiotics or prebiotics. Antibiotics will be used to suppress the undesirable effects of microbiome. Probiotics will introduce the microbial components which are beneficial to humans while prebiotics will be used to enhance the proliferation of these beneficial microbes.

Diverse microbial community like bacteria, viruses, fungi and protozoa are associated with all higher animals. Microorganisms cover most of the host mucosal surfaces, but reside mainly within the gastrointestinal tract. The number of bacteria increase progressively as we move down the gastrointestinal tract from proximal to distal gut with maximum number and diversity in the colon (the stomach contains 10 microbial cells, the duodenum $10^{3}$ cells, the jejunum $10^{4}$ cells, the ileum $10^{7}$ cells and the colon up to $10^{12}$ cells per gram of content). Obligate anaerobic bacteria constitute majority of the gut flora and outnumber aerobic and facultative anaerobes by 100 to 1000 fold. Intestinal microbiota has been implicated in the pathogenesis of several disorders including obesity, diabetes, autism, myasthenia gravis and rheumatoid arthritis.

Probiotics have shown promising results in the treatment and in reducing the risk of various inflammatory and immunological disorders. In neonatal age group, as discussed in a review article in this journal, probiotics are found to be useful in the treatment of infantile colic and have been found to reduce the risk of necrotizing enterocolitis. In children, it has been found to be useful in the treatment of acute infectious diarrhea and inflammatory bowel syndrome (IBS) like symptoms and also reduce the risk of antibiotic associated diarrhea, lactose maldigestion, upper respiratory tract infection and atopic dermatitis. In adults, probiotics are helpful in treatment of IBS like symptoms and vaginal infections, while they reduce the risk of lactose maldigestion, pouchitis, ulcerative colitis and travelers' diarrhea.

According to a recent Cochrane review, E. coli strain Nissle 1917 was found to be equivalent to Mesalamine in the maintenance of remission in patient of mild to moderate ulcerative colitis. Probiotics have further shown to be effective in maintenance of remission in patients of pouchitis already treated by medical or surgical therapy. Italian cohort of over 45,000 volunteers, with a 12 years follow up, reported a reduction in colorectal carcinoma with high intake of yoghurt, although this study did not contain any comparative group. Probiotics have been tried in prevention of allergic disorders but evidence of its efficacy is not convincing enough to recommend routine use of probiotics by the pediatric organizations.

Fecal microbiota transplantation is the process of transplantation of bacteria from healthy individual into a recipient for treatment of various diseases. Fecal transplantation from lean donors to patients of metabolic syndromes resulted in marked reduction in fasting triglycerides with increased peripheral and hepatic insulin sensitivity after 6 weeks. Gastrointestinal tract disturbance like constipation and diarrhea in Parkinsonism and myoclonic dystronia when treated by antibiotics e.g. vancomycin, metronidazole have not only improved GI symptoms but neurological problems also have resolved suggesting a gut brain connection and microbiota as its epicentre.

Prolonged remission of idiopathic thrombocytopenia and symptomatic improvement in chronic fatigue syndrome with fecal microbiota transplantation further encourages us to study the role of microbiota in the gut neural axis. 
Reducing the risk of disease with probiotics is promising but validated biomarkers for many target diseases are lacking. Identifying subjects on the edges of normal physiological range and intervening may be an important approach for future research.

\section{RECOMMENDED READING}

1. Preidis $\mathrm{GA}_{2}$ Versalovic J. Targeting the human microbiome with antibiotics, probiotics and prebiotics: gastroenterology enters the metagenomics era. Gastroenterology 2009;136(6):2015-31.

2. Friedman G. Preface: clinical applications of probiotics in gastroenterology: questions and answers. Gastroenterol Clin North Am 2012;41(4); ix-xii.

3. Naidoo K, Gordon M, Fagbemi AO, Thomas AG, Akobeng AK. Probiotics for maintenance of remission in ulcerative colitis. Cochrane Database Syst Rev 2011;7(12):CD007443.

4. McLean PG, Bergonzelli GE, Collins SM, Bercik P. Targeting the microbiota-gut-brain axis to modulate behavior; which bacterial strain will translate best to humans? Proc Natl Acad Sci USA 2012;109(4):E174. 